Pacific Journal of Mathematic 


\section{TRANSLATION-INVARIANT OPERATORS OF WEAK TYPE}

\section{DANIEL M. OBERLIN}

Let $G$ be a locally compact group and let $m$ be a left Haar measure on $G$. For $0<p<\infty$, let $L^{p}(G)$ be the usual Lebesgue space of functions $f$ on $G$ for which

$$
\|f\|_{p}=\left(\int_{G}|f(x)|^{p} d m(x)\right)^{1 / p}<\infty .
$$

If $T$ is a linear operator which takes $L^{p}(G)$, or a subspace of $L^{p}(G)$, into measurable functions on $G$, then $T$ is said to be of weak type $(p, p)$ if there exists a positive constant $C$ such that

$$
m\{x \in G:|T f(x)| \geqq \alpha\} \leqq C\|f\|_{p}^{p} / \alpha^{p} \text { for } f \in L^{p}(G), \alpha>0 .
$$

We are interested in the translation-invariant operators of weak type $(p, p)$.

To be more precise, for $x \in G$ we define the left and right translation operators $L_{x}$ and $R_{x}$ by $L_{x} f(y)=f(x y)$ and $R_{x} f(y)=f(y x)$ for functions $f$ on $G$ and $y \in G$. An operator $T$ will be called translationinvariant if $T$ commutes with each $R_{x}: T R_{x}=R_{x} T$ for each $x \in G$. We shall prove the following theorems.

THEOREM 1. Suppose that the locally compact group $G$ is amenable. If $0<p<q \leqq 2$ and $T$ is a translation-invariant operator of weak type $(p, p)$ on $L^{p}(G)$, then $T$ is a bounded linear operator on $L^{q}(G)$.

THEOREM 2. Let $G$ be an arbitrary locally compact group and suppose that $0<p<1$. Then $T$ is a translation-invariant operator of weak type $(p, p)$ on $L^{p}(G)$ if and only if $T$ has the form $\sum_{n=1}^{\infty} a_{n} L_{x_{n}}$ for distinct $x_{n} \in G$ and complex numbers $a_{n}$ satisfying $\left|a_{n}\right|=0\left(n^{-1 / p}\right)$.

To state Theorem 3 we need some additional terminology. For a compact group $G$, let $\sum$ denote the dual object of $G$. For $0<$ $p<\infty$ and a subset $E$ of $\sum$, let $L_{E}^{p}\left(=L_{E}^{p}(G)\right)$ denote the closure in $L^{p}(G)$ of the set of trigonometric polynomials with spectrum in $E$.

THEOREM 3. With notation as above, suppose $0<p<q \leqq 2$ and that $T$ is a translation-invariant operator of weak type $(p, p)$ on $L_{E}^{p}$. Then $T$ is bounded on $L_{E}^{q}$. 
Theorem 1 should be compared with a previous result of $M$. Cowling [2]. Cowling's result states that if $T$ is a continuous translation-invariant operator between two rearrangement-invariant Banach function spaces on $G$, then $T$ is automatically bounded on $L^{2}(G)$. We note that the hypothesis of amenability is necessary to Theorem 1: N. Lohoue has proved that for $1<p<2$ there are translationinvariant linear operators bounded on $L^{p}(\mathrm{SL}(2, R))$ which are not bounded on $L^{2}(\mathrm{SL}(2, R))$ [5].

Theorem 2 is an analogue of the result of [7] for operators of weak type. For the circle group $T$, Theorem 2 was established in [8]. But the methods of [8] do not seem to generalize beyond the case of compact $G$.

Theorem 3 is a partial answer to question (ii) of [6]. We mention that if $2<q<p=2 m(m=2,3, \cdots)$, a translation-invariant operator on $L_{E}^{p}$ may fail to be bounded on $L_{E}^{q}$ [1].

2. The proofs. We begin with some preliminaries from probability theory. Our probability space will be the unit interval $I$ equipped with Lebesgue measure, which we shall denote by $P$.

Fix $q$ with $0<q \leqq 2$. A complex-valued random variable $g$ on $I$ is said to be $q$-stable of type $k>0$ if its characteristic function $\chi_{g}(z)=\int_{I} \exp (-i \operatorname{Re}[z \overline{g(t)}]) d P(t)$ is equal to $\exp \left(-k^{q}|z|^{q}\right)(z \in C)$. Now suppose that $\left\{g_{i}\right\}_{i=1}^{\infty}$ is a sequence of independent $q$-stable random variables of type 1 defined on $I$. We shall need the facts that given $n$ and complex numbers $c_{1}, \cdots, c_{n}$,

$$
c_{1} g_{1}+\cdots+c_{n} g_{n} \text { is } q \text {-stable of type }\left(\sum_{1}^{n}\left|c_{i}\right|^{q}\right)^{1 / q},
$$

and

$$
\int_{I}\left|\sum_{1}^{n} c_{i} g_{i}(t)\right|^{p} d P(t)=\left(\sum_{1}^{n}\left|c_{i}\right|^{q}\right)^{p / q} \int_{I}\left|g_{1}(t)\right|^{p} d P(t), \quad 0<p<q .
$$

Lemma 1. For fixed $q$ with $0<q \leqq 2$ there exists a decreasing nonnegative function $\phi_{q}$ defined on $(0, \infty)$ such that if $g$ is a $q$-stable random variable of type $k$ on $I$, then

$$
P\{t \in I:|g(t)| \geqq \alpha\}=\phi_{q}\left(\alpha^{q} / k^{q}\right) .
$$

Proof. This follows from the fact that $g / k$ is $q$-stable of type 1 if $g$ is $q$-stable of type $k$.

Our next lemma is a result for operators of weak type analogous to Lemma 2 of [4]. 
Lemma 2. Fix $p$ and $q$ with $0<p<q \leqq 2$. Let $T$ be a linear operator of weak type $(p, p)$ on a subspace $S$ of $L^{p}(G)$. There exists a positive constant $C$ such that the following holds: If $f(x, y)$ is a continuous function of compact support on $G \times G$ such that $f(\cdot, y) \in S$ for each $y \in G$, then, for $\alpha>0$,

$$
\begin{aligned}
m\{x & \left.\in G:\left(\int_{G}|T f(\cdot, y)(x)|^{q} d m(y)\right)^{1 / q} \geqq \alpha\right\} \\
& \leqq C \int_{G}\left(\int_{G}|f(x, y)|^{q} d m(y)\right)^{p / q} d m(x) / \alpha^{p}
\end{aligned}
$$

Proof. For each $n=1,2, \cdots$ there exist $m(=m(n))$ pairwise disjoint Borel sets $E_{1}, \cdots, E_{m} \subseteq G$ and continuous compactly-supported functions $k_{1}, \cdots, k_{m} \in S$ such that if $\chi_{i}$ is the characteristic function of $E_{i}$ and if

$$
f_{n}(x, y)=\sum_{1}^{m} k_{i}(x) \chi_{i}(y)
$$

then

$$
\begin{aligned}
& \text { support }\left(f_{n}\right) \subseteq K \text { for some compact } K \cong G \text { and all } n \text {, and } \\
& \sup \left\{\left|f_{n}(x, y)-f(x, y)\right|:(x, y) \in G \times G\right\}=o\left(n^{-1}\right) .
\end{aligned}
$$

In the following, $C$ will denote a positive constant which is independent of $f$ but may increase from line to line. The hypothesis on $T$ implies that $C$ may be chosen large enough to insure that

$$
\begin{aligned}
& m\left\{x \in G:\left|T f(\cdot, y)(x)-T f_{n}(\cdot, y)(x)\right| \geqq \alpha\right\} \\
& \quad \leqq C \int_{G}\left|f(x, y)-f_{n}(x, y)\right|^{p} d m(x) / \alpha^{p} \quad(y \in G, \alpha>0) .
\end{aligned}
$$

Integrating this inequality over $G$ with respect to $y$, applying Fubini's theorem, and taking into account (4), we find that

$$
m \times m\left\{(x, y) \in G \times G:\left|T f(\cdot, y)(x)-T f_{n}(\cdot, y)(x)\right| \geqq n^{-1}\right\} \longrightarrow 0 .
$$

It follows that, by passing to a subsequence if necessary, we can assume $T f_{n}(\cdot, y)(x) \rightarrow T f(\cdot, y)(x)$ almost everywhere on $G \times G$. Thus, by Fatou's lemma,

$$
\begin{aligned}
& \underline{\lim } \int_{G}\left|T f_{n}(\cdot, y)(x)\right|^{q} d m(y) \geqq \int_{G}|T f(\cdot, y)(x)|^{q} d m(y) \text { for almost } \\
& \quad \text { all } x \in G .
\end{aligned}
$$

Let $\phi_{q}$ be the function in Lemma 1 and let $\alpha, \beta>0$ be arbitrary. Since $\phi_{q}$ is decreasing, it follows from the inequality above and another application of Fatou's lemma that 


$$
\begin{aligned}
\int_{G} \phi_{q} & \left(\beta^{q} / \int_{G}|T f(\cdot, y)(x)|^{q} d m(y)\right) d m(x) \\
& \leqq\left.\operatorname{li} \rightsquigarrow\right|_{J_{G}} \phi_{q}\left(\beta^{q} / \int_{G}\left|T f_{n}(\cdot, y)(x)\right|^{q} d m(y)\right) d m(x) .
\end{aligned}
$$

Fix a number $M>0$ such that $\phi_{q}\left(M^{-q}\right)>0$. Then

$$
\int_{G}|T f(\cdot, y)(x)|^{q} d m(y) \geqq \alpha^{q}
$$

implies

$$
\phi_{q}\left([\alpha / M]^{q} / \int_{G}|T f(\cdot, y)(x)|^{q} d m(y)\right) \geqq \phi_{q}\left(M^{-q}\right) .
$$

With $\beta=\alpha / M$ in (5) it follows that

$$
\begin{aligned}
& m\left\{x \in G: \int_{G}|T f(\cdot, y)(x)|^{q} d m(y) \geqq \alpha^{q}\right\} \\
& \left.\quad \leqq\left[\phi_{q}\left(M^{-q}\right)\right]^{-1} \underline{\lim } \int_{G} \phi_{q}(\alpha / M)^{q} / \int_{G}\left|T f_{n}(\cdot, y)(x)\right|^{q} d m(y)\right) d m(x),
\end{aligned}
$$

and so (3) will be established when we show

$$
\begin{aligned}
& \underline{\lim } \int_{G} \phi_{q}\left(\beta^{q} / \int_{G}\left|T f_{n}(\cdot, y)(x)\right|^{q} d m(y)\right) d m(x) \\
& \leqq C \beta^{-p} \int_{G}\left(\int_{G}|f(x, y)|^{q} d m(y)\right)^{p / q} d m(x) .
\end{aligned}
$$

To this end, suppose that $h_{1}, \cdots, h_{m}$ are functions in $S$ and that $g_{1}, \cdots, g_{m}$ are independent $q$-stable random variables on $I$ of type 1 . For each $t \in I$ we have

$$
m\left\{x \in G:\left|\sum_{1}^{m} g_{i}(t) T h_{i}(x)\right| \geqq \beta\right\} \leqq C \beta^{-p} \int_{G}\left|\sum_{1}^{m} g_{i}(t) h_{i}(x)\right|^{p} d m(x) .
$$

Integrating this over $I$, using Fubini's theorem, and recalling (2), we find that

$$
\begin{gathered}
\int_{G} P\left\{t \in I:\left|\sum_{1}^{m} g_{i}(t) T h_{i}(x)\right| \geqq \beta\right\} d m(x) \\
\leqq C \beta^{-p} \int_{G}\left(\sum_{1}^{m}\left|h_{i}(x)\right|^{q}\right)^{p / q} d m(x) .
\end{gathered}
$$

For fixed $x \in G$, (1) implies that $\sum_{1}^{m} g_{i}(t) T h_{i}(x)$ is symmetric $q$-stable of type $\left(\sum_{1}^{m}\left|T h_{i}(x)\right|^{q}\right)^{1 / q}$. Thus Lemma 1 and (7) yield

$$
\int_{G} \phi_{q}\left(\beta^{q} / \sum_{1}^{m}\left|T h_{i}(x)\right|^{q}\right) d m(x) \leqq C \beta^{-p} \int_{G}\left(\sum_{1}^{m}\left|h_{i}(x)\right|^{q}\right)^{p / q} d m(x) .
$$

Now (6) follows from (4) and the representation 


$$
f_{n}(x, y)=\sum_{1}^{m} k_{i}(x) \chi_{i}(y)
$$

LEMMA 3. Fix $p$ and $q$ with $0<p<q \leqq 2$. Let $S$ be a subspace of $L^{p}(G)$ such that $R_{x} S \subseteq S$ for each $x \in G$ and let $T$ be a translationinvariant operator of weak type $(p, p)$ on $S$. There exists a positive constant $C$ such that the following holds: Fix a compact symmetric $K \subseteq G$ and a nonvoid compact set $U \subseteq G$. Suppose $u$ is a compactly supported con tinuous function such that $u=1$ on $K K U$. Suppose $h \in S$ is a continuous function supported in $K$ such that

$$
u \cdot\left(R_{y} h\right) \in S, \quad y \in G .
$$

Then

$$
\left(\int_{K}|T h(y)|^{q} d m(y)\right)^{p / q} \leqq C \int_{G}|u(x)|^{p} d m(x)\left(\int_{G}|h(y)|^{q} d m(y)\right)^{p / q} / m(U) .
$$

Proof. Let $V=(K U)^{-1}$. By the translation-invariance of $T$ we have, for arbitrary $x \in G$,

$$
\int_{V}\left|T(u(\cdot) h(\cdot y))(x)_{\mid}^{\mid q}\right|^{q} d m(y)=\int_{V}\left|T\left(u\left(\cdot y^{-1}\right) h(\cdot)\right)(x y)\right|^{q} d m(y) .
$$

Since $y \in V$ implies $u\left(\cdot y^{-1}\right)=1$ on the support of $h$, it follows that the latter integral is

$$
\int_{V}|T h(x y)|^{q} d m(y)=\int_{G}\left|T h(y) \chi_{V}\left(x^{-1} y\right)\right|^{q} d m(y) .
$$

Here $\chi_{V}$ denotes the characteristic function of the set $V$. Now if $x \in U$, then $\chi_{V}\left(x^{-1} y\right)=1$ as long as $y \in K=K^{-1}$. Thus, for $x \in U$,

$$
\int_{K}|T h(y)|^{q} d m(y) \leqq \int_{G}\left|T h(y) \chi_{V}\left(x^{-1} y\right)\right|^{q} d m(y) .
$$

Together with (9) and (10) this gives

$$
\left(\int_{K}|T h(y)|^{q} d m(y)\right)^{1 / q} \leqq\left(\int_{G}|T(u(\cdot) h(\cdot y))(x)|^{q} d m(y)\right)^{1 / q}
$$

if $x \in U$. It follows that

$$
\begin{aligned}
& m\{x \in G:\left(\int_{G}|T(u(\cdot) h(\cdot y))(x)|^{q} d m(y)\right)^{1 / q} \\
&\left.\geqq\left(\int_{K}|T h(y)|^{q} d m(y)\right)^{1 / q}\right\} \geqq m(U) .
\end{aligned}
$$

On the other hand, Lemma 2 (with $f(x, y)=u(x) h(x y)$ and $\alpha=$ $\left.\left(\int_{K}|T h(y)|^{q} d m(y)\right)^{1 / q}\right)$ implies that the LHS of (11) is 


$$
\leqq C \int_{G}\left(\int_{G}|u(x) h(x y)|^{q} d m(y)\right)^{p / q} d m(x) /\left(\int_{K}|T h(y)|^{q} d m(y)\right)^{p / q} .
$$

That is,

$$
m(U) \leqq C \int_{G}|u(x)|^{q} d m(x)\left(\int_{G}|h(y)|^{q} d m(y)\right)^{p / q} /\left(\int_{K}|T h(y)|^{q} d m(y)\right)^{p / q},
$$

which completes the proof of the lemma.

Proof of Theorem 1. Let $h$ be any continuous compactly-supported function on $G$, and let $K$ be any compact symmetric subset of $G$ containing the support of $h$. A characteristic property of amenable groups [3] implies that there exists a compact subset $U$ of $G$ with $m(K K U) / m(U)<2$. It follows that there exists a continuous compactly-supported function $u$ on $G$ with $u=1$ on $K K U$ and $\int_{G}|u(x)|^{p} d m(x) / m(U)<2$. Taking $S=L^{p}(G)$ in Lemma 3 (it is obvious that (8) is satisfied) we conclude that

$$
\left(\int_{K}|T h(y)|^{q} d m(y)\right)^{p / q} \leqq 2 C\left(\int_{G}|h(y)|^{q} d m(y)\right)^{p / q} .
$$

Since $K$ can be any compact symmetric subset of $G$ containing the support of $h$, it follows that $\|T h\|_{q}^{p} \leqq 2 C\|h\|_{q}^{p}$. Since $h$ is an arbitrary continuous compactly-supported function on $G$, the theorem follows.

Proof of Theorem 3. We apply Lemma 3 with $S=L_{E}^{p}$ and $K=$ $U=G$. Then $u=1$ on $G$ and so (8) is satisfied for any continuous $h \in S$. Since such $h$ are dense in $L_{E}^{q}$, Theorem 3 follows immediately from the conclusion of Lemma 3 .

To establish Theorem 2 we require two more lemmas.

LEMMA 4. Let $G$ be a locally compact group. Let $V \cong G$ be a measurable set with $0<m(V) \leqq 1$, and fix $r$ with $0<r<1$. Given a positive number $C_{1}$ there exists another positive number $C_{2}$ such that if $F$ is a nonnegative measurable function on $G$ satisfying

$$
m\left\{x \in G: \int_{G} F(y) \chi_{V}\left(y^{-1} x\right) d m(y) \geqq \alpha\right\} \leqq C_{1} / \alpha^{r} \quad(\alpha>0),
$$

then

$$
\int_{G} F(y) d m(y) \leqq C_{2} .
$$

Proof. Choose nonnegative measurable functions $F_{n}$ on $G$ with $F_{n} \uparrow F$ and $\int_{G} F_{n}(x) d m(x)=a_{n}<\infty$. Write

$$
H(x)=F * \chi_{V}(x)=\int_{G} F(y) \chi_{V}\left(y^{-1} x\right) d m(y)
$$


and, similarly, $H_{n}=F_{n}^{*} \chi_{V}$. Then $H_{n} \leqq H$, so $m\left\{x: H_{n}(x) \geqq \alpha\right\} \leqq C_{1} / \alpha^{r}$ by hypothesis. Also $H_{n} \leqq a_{n}$, so

$$
\begin{aligned}
a_{n} m(V) & =\int_{G} F_{n} * \chi_{V}(x) d m(x)=\int_{G} H_{n}(x) d m(x)=\int_{0}^{a_{n}} m\left\{x: H_{n}(x) \geqq \alpha\right\} d \alpha \\
& \leqq \int_{0}^{a_{n}} C_{1} \alpha^{-r} d \alpha=C_{1} \alpha_{n}^{1-r} /(1-r) .
\end{aligned}
$$

Thus

$$
a_{n} \leqq\left[C_{1} / m(V)(1-r)\right]^{1 / r}=C_{2},
$$

and so

$$
\int_{G} F(y) d m(y) \leqq C_{2}
$$

also.

LEMMA 5. Let $\left\{f_{n}\right\}_{n=1}^{\infty}$ be a sequence of nonnegative measurable functions on $G$ having the same distribution function $F(\alpha)=$ $m\left\{x \in G:\left|f_{n}(x)\right| \geqq \alpha\right\}(\alpha>0)$. Fix $p$ with $0<p<1$. Then if $\alpha>0$ we have

$$
m\left\{x \in G: \sum_{1}^{\infty} n^{-1 / p} f_{n}(x) \geqq \alpha\right\} \leqq C\left\|f_{1}\right\|_{p}^{p} / \alpha^{p},
$$

where $C$ is a constant depending only on $p$.

Proof. Let $C$ denote a positive constant depending only on $p$, but which may increase from line to line. Fix $\alpha>0$. For $n=$ $1,2, \cdots$ let $\chi_{n}$ be the characteristic function of the set

$$
\left\{x \in G: f_{n}(x)>\alpha n^{1 / p}\right\}
$$

and let $\chi_{n}^{\prime}$ be the characteristic function of $\left\{x \in G: f_{n}(x) \leqq \alpha n^{1 / p}\right\}$. We will establish (14) by estimating separately the two quantities

$$
\begin{aligned}
& m\left\{x \in G: \sum_{1}^{\infty} n^{-1 / p} f_{n}(x) \chi_{n}(x) \geqq \alpha\right\} \quad \text { and } \\
& m\left\{x \in G: \sum_{1}^{\infty} n^{-1 / p} f_{n}(x) \chi_{n}^{\prime}(x) \geqq \alpha\right\} .
\end{aligned}
$$

We have

$$
\begin{aligned}
m\{x & \left.\in G: \sum_{1}^{\infty} n^{-1 / p} f_{n}(x) \chi_{n}(x) \geqq \alpha\right\} \leqq \sum_{1}^{\infty} m\left\{x \in G: f_{1}(x)>\alpha n^{1 / p}\right\} \\
& =\alpha^{-p} \sum_{1}^{\infty} \alpha^{p} n m\left\{x \in G: \alpha n^{1 / p}<f_{1}(x) \leqq \alpha(n+1)^{1 / p}\right\} \leqq \alpha^{-p}\left\|f_{1}\right\|_{p}^{p} .
\end{aligned}
$$

To estimate (15) we begin by writing $H(\lambda)=F\left(\lambda^{1 / p}\right)$, so that 
$\left\|f_{n}\right\|_{p}^{p}=-\int_{0}^{\infty} \lambda d H(\lambda)$ for each $n$. Then

$$
\begin{gathered}
\int_{G} \sum_{1}^{\infty}(n+1)^{-1 / p} f_{n}(x) \chi_{n}^{\prime}(x) d m(x)=\sum_{1}^{\infty}(n+1)^{-1 / p} \int_{\left\{f_{n}(x) \leqq \alpha^{1} p^{1}\right\}} f_{n}(x) d m(x) \\
=-\sum_{1}^{\infty}(n+1)^{-1 / p} \int_{0}^{n \alpha^{p}} \lambda^{1 / p} d H(\lambda) \leqq-\int_{1}^{\infty} y^{-1 / p} \int_{0}^{y \alpha^{p}} \lambda^{1 / p} d H(\lambda) d y \\
=-\int_{1}^{\infty} y^{-1 / p} \int_{0}^{\alpha^{p}} \lambda^{1 / p} d H(\lambda) d y-\int_{\alpha^{p}}^{\infty} \lambda^{1 / p} \int_{\lambda / \alpha p}^{\infty} y^{-1 / p} d y d H(\lambda) .
\end{gathered}
$$

Now (15) is

$$
\leqq C \alpha^{-1} \int_{G} \sum_{1}^{\infty}(n+1)^{-1} f_{n}(x) \chi_{n}^{\prime}(x) d m(x),
$$

so, by (16), it suffices to establish

$$
-\alpha^{-1} \int_{1}^{\infty} y^{-1 / p} \int_{0}^{\alpha^{p}} \lambda^{1 / p} d H(\lambda) d y \leqq C\left\|f_{1}\right\|_{p}^{p} / \alpha^{p}
$$

and

$$
-\alpha^{-1} \int_{\alpha p}^{\infty} \lambda^{1 / p} \int_{\lambda / \alpha p}^{\infty} y^{-1 / p} \quad \text { dy } \quad d H(\lambda) \leqq C\left\|f_{1}\right\|_{p}^{p} / \alpha^{p} .
$$

For (17) we note that

$$
-\int_{0}^{\alpha^{p}} \lambda^{1 / p} d H(\lambda)=\int_{\left.\mid f_{1}(x) \leqq \alpha\right\}} f_{1}(x) d m(x)
$$

and

$$
\alpha^{-1} \int_{\left\{f_{1}(x) \leqq \alpha\right\}} f_{1}(x) d m(x) \leqq \alpha^{-p} \int_{\left\{f_{1}(x) \leqq \alpha\right\}} f_{1}^{p}(x) d m(x) .
$$

Since $\int_{1}^{\infty} y^{-1 / p} d y<\infty$, this establishes (17). On the other hand

$$
\int_{\lambda / \alpha p}^{\infty} y^{-1 / p} d y=\left(p^{-1}-1\right) \lambda^{1-1 / p} \alpha^{1-p}
$$

Thus

$$
-\alpha^{-1} \int_{\alpha^{p}}^{\infty} \lambda^{1 / p} \int_{\lambda / \alpha p}^{\infty} y^{-1 / p} d y d H(\lambda) \leqq-C \alpha^{-p} \int_{\alpha^{p}}^{\infty} \lambda d H(\lambda) \leqq C\left\|f_{1}\right\|_{p}^{p} / \alpha^{p} .
$$

This is (18) and so the proof of the lemma is complete.

Proof of Theorem 2. The "if" part of Theorem 2 is an immediate consequence of Lemma 5 . So suppose $T$ is a translation-invariant operator of weak type $(p, p)$ on $L^{p}(G)(0<p<1)$, and we will show that $T$ has the form $\sum_{n=1}^{\infty} a_{n} L_{x_{n}},\left|a_{n}\right|=0\left(n^{-1 / p}\right)$. Fix $q$ with $0<p<$ 
$q \leqq 2$. We will begin by showing that $T$ is "locally bounded" on $L^{q}(G)$.

Let $U$ and $V$ be neighborhoods of the identity in $G$ with $U$ relatively compact, $V$ symmetric, $V^{2} \leqq U$, and $m(V) \leqq 1$. Let $u$ be a continuous function with compact support satisfying $u(x)=1$ for $x \in U$, and let $h$ be an arbitrary continuous function with support contained in $V$. According to Lemma 2, where we take $S=L^{p}(G)$ and $f(x, y)=u(x) h(x y)$, we have

$$
\begin{aligned}
m\{x & \left.\in G:\left(\int_{G}|T(u(\cdot) h(\cdot y))(x)|^{q} d m(y)\right)^{1 / q} \geqq \beta\right\} \\
& \leqq C \int_{G}|u(x)|^{p} d m(x)\left(\int_{G}|h(y)|^{q} d m(y)\right)^{p / q} / \beta^{p} \quad(\beta>0) .
\end{aligned}
$$

Since $T$ is translation-invariant,

$$
\int_{V}|T(u(\cdot) h(\cdot y))(x)|^{q} d m(y)=\int_{V}\left|T\left(u\left(\cdot y^{-1}\right) h(\cdot)\right)(x y)\right|^{q} d m(y) .
$$

Since $V^{2} \subseteq U, V$ is symmetric, and $h$ is supported in $V$, it follows that $u\left(\cdot y^{-1}\right)$ is equal to 1 on the support of $h$ as long as $y \in V$. Thus the last integral is equal to

$$
\int_{V}|T h(x y)|^{q} d m(y)=\int_{G}|T h(y)|^{q} \chi_{V}\left(y^{-1} x\right) d m(y),
$$

where we have used $V=V^{-1}$. Thus

$$
\int_{G}|T h(y)|^{q} \chi_{V}\left(y^{-1} x\right) d m(y) \leqq \int_{G}|T(u(\cdot) h(\cdot y))(x)|^{q} d m(y) .
$$

With (19) (where we substitute $\alpha$ for $\beta^{q}$ ) we have

$$
\begin{aligned}
m\{x & \left.\in G: \int_{G}|T h(y)|^{q} \chi_{V}\left(y^{-1} x\right) d m(y) \geqq \alpha\right\} \\
& \leqq C \int_{G}|u(x)|^{p} d m(x)\left(\int_{G}|h(y)|^{q} d m(y)\right)^{p / q} / \alpha^{p / q} .
\end{aligned}
$$

Taking $r=p / q, C_{1}=C \int_{G}|u(x)|^{p} d m(x)$, and $F(y)=|T h(y)|^{q}$ in Lemma 4, we see that $\|h\|_{q}^{q} \leqq 1$ implies $\|T h\|_{q}^{q} \leqq C_{2}$ for some fixed positive number $C_{2}$ and any continuous $h$ supported in $V$. It follows that

$$
\|T h\|_{q}^{q} \leqq C_{2}\|h\|_{q}^{q}
$$

holds for any measurable $h$ supported in $V$. (Thus $T$ is "locally bounded" on $L^{q}(G)$.)

If $0<p<q<1$, it follows from (20), from the translationinvariance of $T$, and from the subadditivity of $\|\cdot\|_{q}^{q}$ that $T$ is actually bounded on $L^{q}(G)$. Now the theorem in [6] shows that $T$ has the 
form $\sum_{1}^{\infty} a_{n} L_{x_{n}}$ for distinct $x_{n} \in G$ and numbers $a_{n}$ satisfying $\sum_{1}^{\infty}\left|a_{n}\right|^{q}<$ $\infty$. Using the fact that $T$ is actually of weak type $(p, p)$, it is easy to see that

$$
\operatorname{card}\left\{n:\left|a_{n}\right| \geqq \alpha\right\}=0\left(\alpha^{-p}\right) \quad(\alpha>0) .
$$

Thus if $\left\{\left|a_{n}^{*}\right|\right\}_{n=1}^{\infty}$ is a decreasing rearrangement of the sequence $\left\{\left|a_{n}\right|\right\}_{n=1}^{\infty}$, it follows that $\left|a_{n}^{*}\right|=0\left(n^{-1 / p}\right)$. This completes the proof of Theorem 2.

\section{REFERENCES}

1. W. R. Bloom, Interpolation of multipliers of $L_{\gamma}^{p}$, to appear.

2. M. Cowling, Some applications of Grothendieck's theory of topological tensor products in harmonic analysis, Math. Ann., 232 (1978), 273-285.

3. W. Emerson and F. Greenleaf, Covering properties and Folner conditions for locally compact groups, Math. Zeit., 102 (1967), 370-384.

4. C. Herz and N. Rivière, Estimates for translation-invariant operators on spaces with mixed norms, Studia Math., 44 (1972), 511-515.

5. N. Lohoué, Estimation $L^{p}$ des coefficients de certaines représentations et opérateurs de convolution, to appear in Advances in Mathematics.

6. D. Oberlin, Multipliers of $L_{E}^{p}, I I$, Studia Math., 59 (1977), 235-248.

7. - Translation-invariant operators on $L^{p}(G), 0<p<1(I I)$, Canad. J. Math., 29 (1977), 626-630.

8. S. Sawyer, Maximal inequalities of weak type, Ann of Math., 84 (1966), 157-174.

Received October 16, 1978. Partially supported by NSF Grant MCS 76-02267-A01.

The Florida State University

TAllahassee, F1 32306 


\section{PACIFIC JOURNAL OF MATHEMATICS}

\section{EDITORS}

DONALD BABBITT (Managing Editor)

University of California

Los Angeles, California 90024

HUgo RossI

University of Utah

Salt Lake City, UT 84112

C. C. MOORE and ANDREW OGG

University of California

Berkeley, CA 94720

\section{J. DUGUNDJI}

Department of Mathematics University of Southern California Los Angeles, California 90007

R. Finn and J. Milgram Stanford University Stanford, California 94305

\section{ASSOCIATE EDITORS}

E. F. BECKENBACH

B. H. NEUMANN

F. WOLF

K. YoshidA

\section{SUPPORTING INSTITUTIONS}

UNIVERSITY OF BRITISH COLUMBIA CALIFORNIA INSTITUTE OF TECHNOLOGY UNIVERSITY OF CALIFORNIA MONTANA STATE UNIVERSITY UNIVERSITY OF NEVADA, RENO NEW MEXICO STATE UNIVERSITY OREGON STATE UNIVERSITY UNIVERSITY OF OREGON
UNIVERSITY OF SOUTHERN CALIFORNIA STANFORD UNIVERSITY UNIVERSITY OF HAWAII UNIVERSITY OF TOKYO UNIVERSITY OF UTAH WASHINGTON STATE UNIVERSITY UNIVERSITY OF WASHINGTON 


\section{Pacific Journal of Mathematics}

Vol. 85, No. $1 \quad$ September, 1979

Ralph Alexander, Metric averaging in Euclidean and Hilbert spaces...... 1

B. Aupetit, Une généralisation du théorème de Gleason-Kahane-Żelazko

pour les algèbres de Banach..............................

Lung O. Chung, Jiang Luh and Anthony N. Richoux, Derivations and

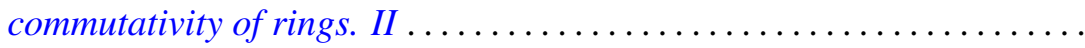

Lynn Harry Erbe, Integral comparison theorems for third order linear

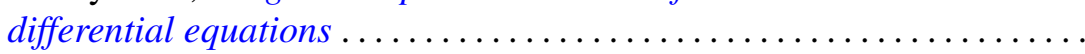

Robert William Gilmer, Jr. and Raymond Heitmann, The group of units of a

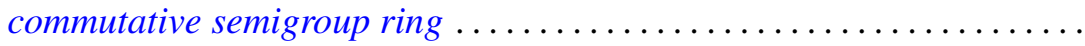

George Grätzer, Craig Robert Platt and George William Sands, Embedding lattices into lattices of ideals ...........................

Raymond D. Holmes and Anthony Charles Thompson, $n$-dimensional area and content in Minkowski spaces ....................... 77

Harvey Bayard Keynes and M. Sears, Modelling expansion in real flows....

Taw Pin Lim, Some classes of rings with involution satisfying the standard

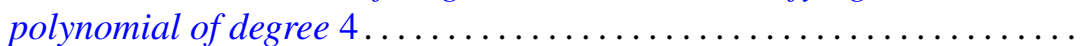

Garr S. Lystad and Albert Robert Stralka, Semilattices having bialgebraic congruence lattices ................................... 131

Theodore Mitchell, Invariant means and analytic actions . . . . . . . . . 145

Daniel M. Oberlin, Translation-invariant operators of weak type ........ 155

Raymond Moos Redheffer and Wolfgang V. Walter, Inequalities involving

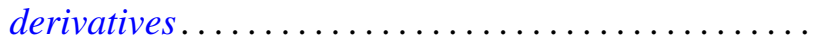

Eric Schechter, Stability conditions for nonlinear products and semigroups ................................

Jan Søreng, Symmetric shift registers ........................ 201

Toshiji Terada, On spaces whose Stone-Čech compactification is $\mathrm{Oz} \ldots \ldots .231$

Richard Vrem, Harmonic analysis on compact hypergroups ... 